\title{
Natural Language Processing utilization in Healthcare
}

\author{
Syihaabul Hudaa, Dwi Bambang Putut Setiyadi, E. Laxmi Lydia, K. Shankar, Phong Thanh \\ Nguyen, Wahidah Hashim, Andino Maseleno
}

\begin{abstract}
The significance of consolidating Natural Language Processing (NLP) techniques in clinical informatics research has been progressively perceived over the previous years, and has prompted transformative advances. Ordinarily, clinical NLP frameworks are created and assessed on word, sentence, or record level explanations that model explicit traits and highlights, for example, archive content (e.g., persistent status, or report type), record segment types (e.g., current meds, past restorative history, or release synopsis), named substances and ideas (e.g., analyses, side effects, or medicines) or semantic qualities (e.g., nullification, seriousness, or fleetingness). While some NLP undertakings consider expectations at the individual or gathering client level, these assignments still establish a minority. Here we give an expansive synopsis and layout of the difficult issues engaged with characterizing suitable natural and outward assessment strategies for NLP look into that will be utilized for clinical results research, and the other way around. A specific spotlight is set on psychological wellness investigate, a zone still generally understudied by the clinical NLP look into network, however where NLP techniques are of prominent importance. Ongoing advances in clinical NLP strategy improvement have been huge, yet we propose more accentuation should be put on thorough assessment for the field to progress further. To empower this, we give noteworthy recommendations, including an insignificant convention that could be utilized when announcing clinical NLP strategy improvement and its assessment.
\end{abstract}

Keywords : NLP, Artificial Intelligence, HER.

\section{INTRODUCTION}

The health care industry is quick understanding the significance of information, gathering data from EHRs, sensors, and different sources. Be that as it may, the battle to comprehend the information gathered in the process may seethe on for a considerable length of time. Since the human

Revised Manuscript Received on July 22, 2019.

* Correspondence Author

Syihaabul Hudaa, Ahmad Dahlan University of Technology and Business, Indonesia. E-mail: hudaasyihaabul@gmail.com

Dwi Bambang Putut Setiyadi, Universitas Widya Dharma Klaten, Indonesia.

E. Laxmi Lydia, Professor, Vignan's Institute of Information Technology(A), Department of Computer Science and Engineering, Visakhapatnam, Andhra Pradesh, India.

K. Shankar*, Department of Computer Applications, Alagappa University, Karaikudi, India. E-mail: shankarcrypto@gmail.com

Phong Thanh Nguyen*, Department of Project Management, Ho Chi Minh City Open University, Vietnam. E-mail: phong.nt@ou.edu.vn

Wahidah Hashim, Institute of Informatics and Computing Energy, Universiti Tenaga Nasional, Malaysia.

Andino Maseleno, Institute of Informatics and Computing Energy, Universiti Tenaga Nasional, Malaysia. services framework has begun embracing front line advancements, there is a tremendous measure of information gathered in storehouses. Medicinal services associations need to digitize forms, yet not superfluously disturb set up clinical work processes. In this manner, we currently have as much as 80 percent of information unstructured and of low quality. This carries us to an appropriate test of information extraction and usage in the health care space through NLP in Healthcare.

This information as it is today, and given the measure of time and exertion it would requirement for people to peruse and reformat it, is unusable. In this way, we can't yet settle on powerful choices in health care through investigation as a result of the structure our information is in. Along these lines, there is a higher need to use this unstructured information as we move from expense for-administration medicinal services model to esteem based consideration.

This is the place Natural Language Processing, a subcategory of Artificial Intelligence can come in. NLP based chatbots as of now have the abilities of well and really imitating human conduct and executing a horde of errands. With regards to actualizing the equivalent on an a lot bigger use case, similar to a clinic - it tends to be utilized to parse data and concentrate basic strings of information, accordingly offering an open door for us to use unstructured information.

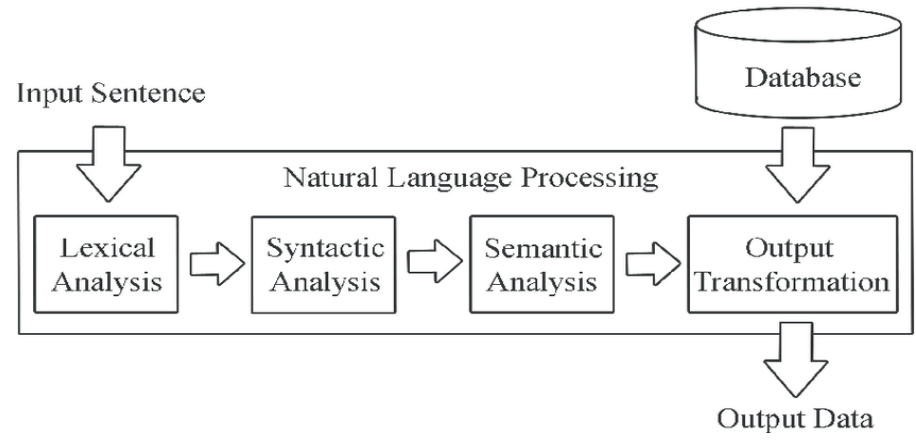

Figure 1: NLP is a core of AI

Streamlining of expense while safeguarding or improving nature of consideration is a focal point of national health change. One noteworthy indicator of mind-boggling expense of treatment is psychological well-being comorbidity-one investigation found that, by and large, 
patients with an emotional wellness finding were 2.2 occasions more costly than patients without a psychological wellness diagnosis. 1 notwithstanding psychological wellness analyze, there are other social medical problems that may not bring about an analysis, yet foresee expanded paces of human services utilization.

This increment in usage is speculated to be because of a diminished capacity to think about self and high paces of pointless care. 3 notwithstanding cost contemplations, the extra medicinal services use related with conduct health conditions may likewise represent the health danger of unintended negative treatment results from superfluous consideration.

\section{NATURAL LANGUAGE PROCESSING}

Natural language preparing is the general term used to depict the way toward utilizing of PC calculations to recognize key components in ordinary language and concentrate importance from unstructured spoken or composed info. NLP is a control of software engineering that requires aptitudes in man-made consciousness, computational semantics, and other AI disciplines.

Still more are fixated on giving information to clients by recognizing and separating key subtleties from colossally enormous collections of data, similar to super-human speed perusers with about boundless memory limit.

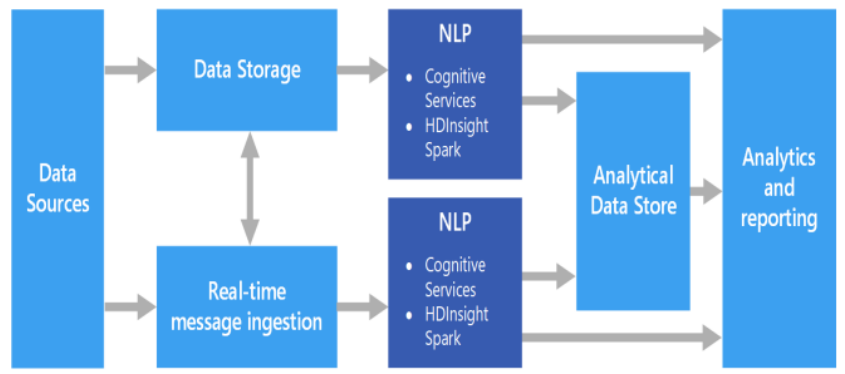

Figure 2: NLP

Explicit undertakings for NLP frameworks may include:

- Summarizing long squares of story content, for example, a clinical note or scholastic diary article, by distinguishing key ideas or expressions present in the source material

- Mapping information components present in unstructured content to organized fields in an electronic health record so as to improve clinical information respectability

- Converting information the other way from machine-coherent arrangements into common language for announcing and instructive purposes

- Answering one of a kind free-content inquiries that require the blend of various information sources

- Engaging in optical character recognition to turn pictures, as PDF records or outputs of consideration synopses and imaging reports, into content documents that would then be able to be parsed and broke down

- Conducting discourse recognition to enable clients to manage clinical notes or other data that would then be able to be transformed into content
Numerous natural language handling frameworks "learn" after some time, reabsorbing the aftereffects of past connections as input about which results were precise and which did not meet desires.

These AI projects can work dependent on likelihood, which gauge the probability that a given bit of information is really what the client has mentioned. In view of whether that answer meets endorsement, the probabilities can be balanced later on to meet the developing needs of the end-client.

\section{FACTORS BEHIND NLP In HealthCARE System}

NLP, a part of AI, goes for fundamentally decreasing the separation between the abilities of a human and a machine. As it getting increasingly more footing in the medicinal services space, providers are concentrating on creating arrangements that can comprehend, break down, and produce languages would humans be able to can get it.

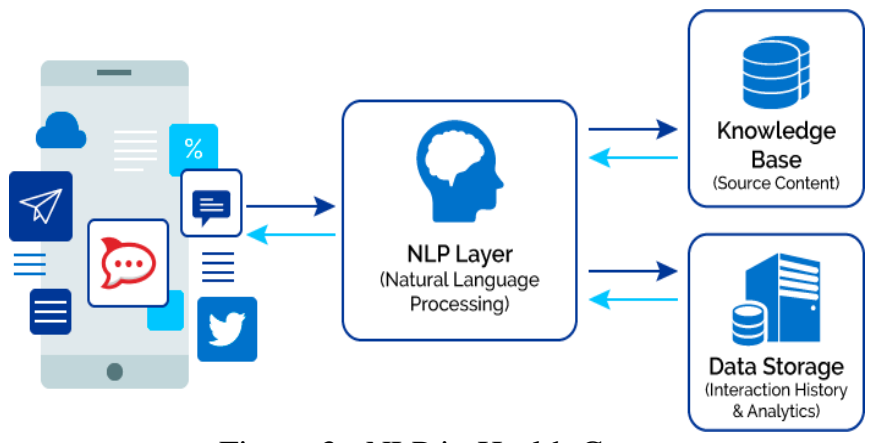

Figure 3 : NLP in Health Care

There is a further requirement for voice recognize frameworks that can consequently react to inquiries from patients and human services clients. There are a lot more drivers of NLP in Healthcare as explained underneath:

- Handle the Surge in Clinical Data

The expanded utilization of patient health record frameworks and the advanced change of medication has prompted a spike in the volume of information accessible with health care associations. The need to bode well out of this information and attract trustworthy bits of knowledge happens to be a noteworthy driver.

- Support Value-Based Care and Population Health Management

The move in plans of action and result desires is driving the requirement for better utilization of unstructured information. Conventional health data frameworks have been concentrating on getting an incentive from the 20 percent of medicinal services information that comes in organized organizations through clinical channels.

For cutting edge understanding health record frameworks, oversaw care, PHM applications, and investigation and revealing, there is a pressing need to take advantage of the supply of unstructured data that is just getting heaped up with medicinal services associations.

NLP in Healthcare could understand these difficulties through various use cases. How about we investigate a few them: 


\section{Improving Clinical Documentation}

Electronic Health Record arrangements regularly have an intricate structure, so that archiving information in them is an issue. With discourse to-content transcription, information can be consequently caught at the purpose of consideration, opening up doctors from the monotonous undertaking of reporting care conveyance.

\section{Making CAC progressively Efficient}

Computer-helped coding can be improved from multiple points of view with NLP. CAC extricates data about strategies to catch codes and expand claims. This can genuinely help HCOs make the move from charge for-administration to a worth based model, along these lines improving the patient experience fundamentally.

\section{-ImprovePatient-Provider Interactions with HER}

Patients these days need full focus from their human services providers. This leaves specialists feeling overpowered and wore out as they bring to the table customized administrations while likewise overseeing troublesome documentation including charging administrations.

Studies have demonstrated how a greater part of consideration experts experience burnout at their working environments. Coordinating NLP with electronic health record frameworks will help take off remaining burden from specialists and make investigation simpler. Effectively, remote helpers, for example, Siri, Cortana, and Alexa have made it into human services associations, filling in as authoritative guides, assisting with client administration errands and help work area obligations.

Before long, NLP in Healthcare may make menial helpers traverse to the clinical side of the health care industry as requesting partners or medicinal copyists.

\section{-Empower Patients with Health Literacy}

With conversational AI previously being a triumph inside the human services space, a key use-case and advantage of executing this innovation is the capacity to enable patients to comprehend their indications and increase more learning about their conditions. By ending up progressively mindful of their health conditions, patients can settle on educated choices, and keep their health on track by interfacing with a clever chatbot.

In a recent report, scientists utilized NLP answers for match clinical terms from their archives with their layman language partners. Thusly, they expected to improve persistent EHR understanding and the patient entryway experience. Common Language Processing in human services could help patients' comprehension of EHR entryways, opening up chances to make them increasingly mindful of their health.

\section{-Address the Need for Higher Quality of Healthcare}

NLP can be the leader in evaluating and improving the nature of medicinal services by estimating doctor execution and distinguishing holes in consideration conveyance.

Research has demonstrated that man-made consciousness in health care can facilitate the procedure of doctor evaluation and robotize tolerant finding, lessening the time and human exertion required in completing routine undertakings, for example, quiet conclusion. NLP in health care can likewise distinguish and moderate potential blunders in consideration conveyance. An investigation demonstrated that NLP could likewise be used in estimating the nature of human services and screen adherence to clinical rules.

\section{-Identify Patients who Need Improved Care}

AI and NLP devices have the abilities expected to recognize patients with complex health conditions who have a background marked by psychological well-being or substance misuse and need improved consideration. Factors, for example, nourishment frailty and lodging precariousness can discourage the treatment conventions, accordingly convincing these patients to bring about more expense in their lifetime.

The information of a patient's economic health and demography is regularly difficult to situate than their clinical data since it is more often than not in an unstructured organization. NLP can help take care of this issue. NLP can likewise be utilized to improve care coordination with patients who have social health conditions. Both, Natural Language Processing and Machine Learning can be used to mine patient information and identify those that are in danger of falling through any holes in the health care framework

Since the medicinal services industry produces both organized and unstructured information, it is critical for health care associations to refine both before executing NLP in human services.

\section{CONCLUSION}

We have tried to give an expansive framework of the ebb and flow state-ofthe-workmanship, openings, difficulties, and needs in the utilization of NLP for health results investigate, with a specific spotlight on assessment strategies. We have laid out methodological viewpoints from a clinical just as a NLP point of view and distinguish three fundamental difficulties: information accessibility, assessment workbenches and detailing measures. In light of these, we give noteworthy direction to each recognized test. We propose an insignificant organized convention that could be utilized when announcing clinical NLP technique improvement and its assessment, to empower straightforwardness and reproducibility. We imagine further advances especially in strategies for information get to, assessment techniques that move past current characteristic measurements and draw nearer to clinical practice and utility, and in straightforward and reproducible strategy improvement.

\section{ACKNOWLEDGMENT}

This article has been written with financial support of RUSA-Phase 2.0 grant sanctioned vide Letter No. F. 24-51/2014-U, Policy (TNMulti-Gen), Dept. of Edn. Govt. of India, Dt. 09.10.2018. 


\section{REFERENCES}

1. Yang, J.-J.; Li, J.; Mulder, J.; Wang, Y.; Chen, S.; Wu, H.; Wang, Q.; Pan, $\mathrm{H}$. Emerging information technologies for enhanced healthcare. Comput. Ind. 2015, 69, 3-11. [CrossRef]

2. Cortada, J.W.; Gordon, D.; Lenihan, B. The Value of Analytics in Healthcare; Report No.: GBE03476-USEN-00; IBM Institute for Business Value: Armonk, NY, USA, 2012.

3. Prokosch, H.-U.; Ganslandt, T. Perspectives for medical informatics. Methods Inf. Med. 2009, 48, 38-44.[CrossRef] [PubMed]

4. Simpao, A.F.; Ahumada, L.M.; Gálvez, J.A.; Rehman, M.A. A review of analytics and clinical informatics inhealth care.J. Med. Syst. 2014, 38, 45. [CrossRef] [PubMed]

5. Ghassemi, M.; Celi, L.A.; Stone, D.J. State of the art review: The data revolution in critical care. Crit. Care2015, 19, 118. [CrossRef] [PubMed]

6. Tomar, D.; Agarwal, S. A survey on Data Mining approaches for Healthcare. Int. J. Bio-Sci. Bio-Technol. 2013,5, 241-266. [CrossRef]

7. Huang, Y.; McCullagh, P.; Black, N.; Harper, R. Feature selection and classification model construction ontype 2 diabetic patients' data. Artif.Intell. Med. 2007, 41, 251-262. [CrossRef] [PubMed]

8. T. Althoff, K. Clark, J. Leskovec, Natural Language Processing for Mental Health:Large Scale Discourse Analysis of Counseling Conversations, CoRR abs/1605.04462.URL<http://arxiv.org/abs/1605.04462>.

9. K. Sparck Jones, Evaluating Natural Language Processing Systems An Analysis andReview, Lecture Notes in Computer Science, Lecture Notes in Artificial Intelligence, 1083, 1995.

10. P. Paroubek, S. Chaudiron, L. Hirschman, Editorial: Principles of Evaluation inNatural Language Processing, TAL 48 (1) (2007) 7-31 http://www.atala.org/ Editorial.

11. L. Dybkjaer, Evaluation of Text and Speech Systems, Text, Speech and LanguageTechnology, 37, 2007.

12. S. Wu, T. Miller, J. Masanz, M. Coarr, S. Halgrim, D. Carrell, C. Clark, Negation's notsolved: generalizability versus optimizability in clinical natural language processing,PLOS One 9 (11) (2014) 1-11, https://doi.org/10.1371/journal.pone.0112774.

13. D. Demner-Fushman, W.W. Chapman, C.J. McDonald, What can natural language processing do for clinical decision support? J. Biomed. Inform. 42 (5) (2009)760-772, https://doi.org/10.1016/j.jbi.2009.08.007.

14. K. Zheng, V.G.V. Vydiswaran, Y. Liu, Y. Wang, A. Stubbs, O. Uzuner, A.E. Gururaj,S. Bayer, J. Aberdeen, A. Rumshisky, S. Pakhomov, H. Liu, $\mathrm{H}$. $\mathrm{Xu}$, Ease of adoptionof clinical natural language processing software: an evaluation of five systems, J.Biomed. Inform. 58 Suppl (2015) S189-96.

15. D.R. Kaufman, B. Sheehan, P. Stetson, A.R. Bhatt, A.I. Field, C. Patel, J.M. Maisel, Natural language processing-enabled and conventional data capture methods forinput to electronic health records: a comparative usability study, JMIR Med. Inform.4 (4) (2016) e35.

16. H. Suominen, L. Zhou, L. Hanlen, G. Ferraro, Benchmarking clinical speech recognitionand information extraction: new data, methods, and evaluations, JMIRMed. Inform. 3 (2) (2015) e19, https://doi.org/10.2196/medinform.4321.

17. E. Aramaki, M. Morita, Y. Kano, T. Ohkuma, Overview of the NTCIR-11 MedNLPtask, in: Proceedings of the 11th NTCIR Conference, NII Testbeds and Communityfor Information access Research (NTCIR), Tokyo, Japan, 2014, pp. 147-154. 